\title{
SÍNTESE E CARACTERIZAÇÃO DO CARBETO DE MOLIBDÊNIO NANOESTRUTURADO
}

\author{
K. K. P. GOMES ${ }^{1}$, C. P. de SOUZA ${ }^{2}$ \\ ${ }^{1}$ Universidade Federal Rural do Semi-Árido, Departamento de Agrotecnologia e Ciências Sociais \\ ${ }^{2}$ Universidade Federal do Rio Grande do Norte, Departamento de Engenharia Química \\ E-mail para contato: kalyanne@ufersa.edu.br
}

\begin{abstract}
RESUMO - A obtenção de carbetos de metais de transição tem recebido uma atenção considerável porque estes materiais tem uma combinação única de propriedades físicas, químicas e mecânicas além de poderem ser usados em aplicações que envolvem condições severas de pressão e temperatura. Neste trabalho o carbeto de molibdênio foi obtido a partir do precursor heptamolibdato de amônio em reator de leito fixo, com uma taxa de aquecimento de $5^{\circ} \mathrm{C} / \mathrm{min}$, em fluxo de metano e hidrogênio numa vazão de $15 \mathrm{~L} / \mathrm{h}$ com $5 \%$ de metano para todos os ensaios. As temperaturas estudadas foram 350, 500, 600, $650,660,675$ e $700^{\circ} \mathrm{C}$ nos tempos de isoterma de $0,60,120$ e 180 minutos, sendo as fases presente, estrutura cristalina e tamanho de cristalitos obtidos por refinamento de estrutura pelo método de Rietveld. $\mathrm{O}$ carbeto de molibdênio obtido a $660^{\circ} \mathrm{C}$ e 3 horas de reação apresentou o menor tamanho de cristalito que foi de $24 \mathrm{~nm}$, sendo caracterizado por MEV, MET, análise elementar e ICP-AES.
\end{abstract}

\section{INTRODUÇÃO}

O estudo da produção e caracterização de materiais ultrafinos e nanoestruturados tem sido um dos temas mais atraentes da pesquisa fundamental e tecnológica nos últimos anos, devido às possibilidades de melhoria das diversas propriedades que os materiais nanoestruturados podem ter em comparação aos materiais obtidos pelos processos convencionais (Patel e Subrahmanyam, 2008). A técnica convencional de preparação dos carbetos, herdada pela industria metalúrgica, envolve a reação do metal, seu hidreto ou o oxido do metal com quantidades apropriadas de carbono. A temperatura de reação é geralmente muito alta, superiores a $1200^{\circ} \mathrm{C}$ para o $\mathrm{Mo}_{2} \mathrm{C}$ (Levy, 1977). Medeiros et al. (2001) apresentaram um método de produção de carbetos de metais refratários (W e $\mathrm{Nb}$ ) em baixa temperatura, a partir de precursores mais reativos, através de reação gás-sólido. Os precursores utilizados na síntese desses carbetos foram o paratungstato de amônio (APT) e o tri(oxalato)oxiniobato de amônio mono-hidratado. Gu et al. (2003) sintetizaram o carbeto de molibdênio com tamanho de cristalito na ordem de $30 \mathrm{~nm}$ via co-redução do sódio do penta-cloreto de molibdênio $\left(\mathrm{MoCl}_{5}\right)$ e tetra-brometo de carbono $\left(\mathrm{CBr}_{4}\right)$ em benzeno numa temperatura de $350^{\circ} \mathrm{C}$, mas foram necessárias 12 horas de reação. Mas recentemente Khabbaz et al. (2013) obtiveram o carbeto de molibdênio com tamanho de cristalito de $54 \mathrm{~nm}$ por moagem de alta energia com uma mistura de $\mathrm{MoO}_{3}$ e carbono. Neste trabalho o carbeto de molibdênio foi obtido a partir do heptamolibdato de molibdênio sendo aquecido em atmosfera controlada de metano e hidrogênio. 


\section{METODOLOGIA}

$\mathrm{O}$ carbeto de molibdênio $\left(\mathrm{Mo}_{2} \mathrm{C}\right)$ foi produzido a partir do precursor heptamolibdato de amônio $\left[\left(\mathrm{NH}_{4}\right)_{6} \mathrm{Mo}_{7} \mathrm{O}_{24} \cdot 4 \mathrm{H}_{2} \mathrm{O}\right]$. O hidrogênio $\left(\mathrm{H}_{2}\right)$ e o metano $\left(\mathrm{CH}_{4}\right)$ foram utilizados como gases redutores e fonte de carbono, respectivamente. As reações entre o precursor e a mistura de metano e hidrogênio foram efetuadas em um forno resistivo bipartido, composto de um reator de leito fixo de alumina com $35 \mathrm{~mm}$ de diâmetro externo e $80 \mathrm{~cm}$ de comprimento. Para acomodar a amostra de precursor no reator de leito fixo foi utilizada uma navícula de $20 \mathrm{~mm}$ de largura, $15 \mathrm{~mm}$ de altura e $80 \mathrm{~mm}$ de comprimento, utilizando para todos os ensaios uma massa de 1,5 gramas, que foi introduzida no tubo de alumina até a parte central do forno, conforme mostra a Figura 1.

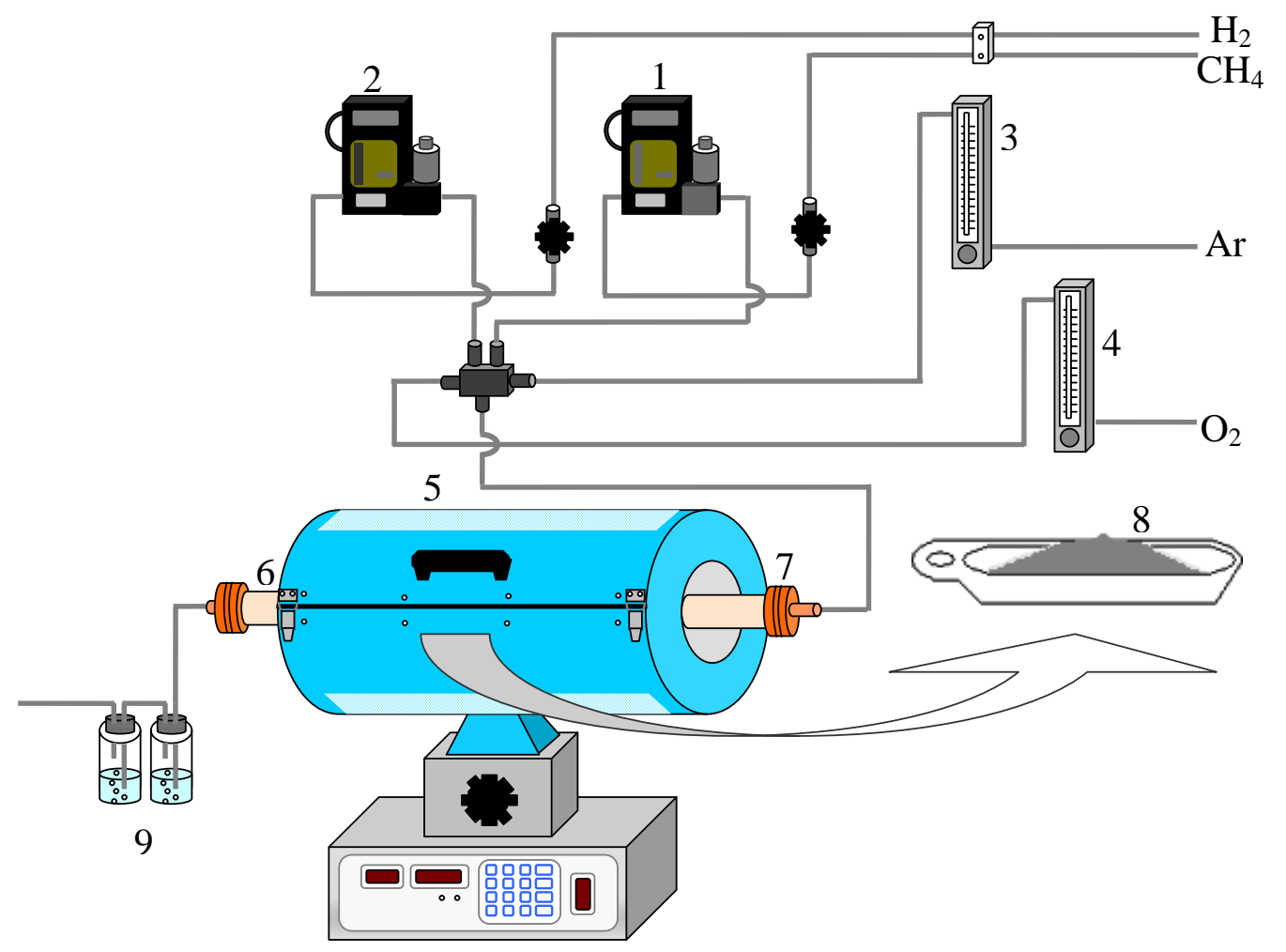

Figura 1 - Forno resistivo bipartido com reator de leito fixo utilizado. 1) Controlador mássico para o gás metano; 2) Controlador mássico para o gás hidrogênio; 3) Rotâmetro do gás argônio; 4) Rotâmetro do gás oxigênio; 5) Forno resistivo bipartido; 6) Reator de leito fixo de alumina; 7)

Flanges para a vedação; 8) Navícula de alumina e amostra do precursor; 9) Borbulhadores.

O carbeto de molibdênio foi obtido através de reações de decomposição-redução-carbonetação nas temperaturas de $350,500,600,650,660,675$ e $700^{\circ} \mathrm{C}$ e nos tempos de isoterma de $0,60,120$ e 180 minutos. A vazão da mistura gasosa usada foi de $15 \mathrm{~L} / \mathrm{h}\left(\mathrm{H}_{2} / \mathrm{CH}_{4}\right)$ com $5 \%$ de metano. Ao final da reação de decomposição-redução-carbonetação, o fluxo de gases reagentes foi trocado por um fluxo de argônio $(10,8 \mathrm{~L} / \mathrm{h})$ e este fluxo foi mantido até a temperatura ambiente. Para o carbeto de 


\section{9 a 22 de outubro de 2014 \\ Florianópolis/SC}

molibdênio obtido a $660^{\circ} \mathrm{C}$ com tempo de isoterma de 3 horas, após ser atingida a temperatura ambiente, o fluxo de argônio foi trocado por um fluxo de $1 \%$ de oxigênio em argônio com vazão total de $10 \mathrm{~L} / \mathrm{h}$ e este fluxo foi mantido por 3 horas e em seguida foi feita a caracterização do carbeto (Bej, et al., 2003; Wang et al., 2005).

A determinação das fases e estruturas cristalinas dos resíduos obtidos nas reações de decomposição-redução-carbonetação foram obtidos pelo programa HighScore da Philips. Os padrões de difração foram submetidos ao refinamento de estrutura Rietveld utilizando o programa DBWS. Os padrões de difração foram obtidos a temperatura ambiente $\left(24^{\circ} \mathrm{C}\right)$ por um difratômetro convencional para amostra policristalinas da marca Rigaku modelo DMAXB operado numa potência de $40 \mathrm{kV} / 25 \mathrm{~mA}$ com radiação incidente de $\mathrm{Cu}$. $\mathrm{O}$ feixe difratado pela amostra foi monocromatizado por um monocromador curvo de grafite. O intervalo angular escolhido foi de $2 \theta: 10-80^{\circ}$ numa varredura contínua com velocidade de $1 / 4 \%$ min e com passo de $0,02^{\circ}$. A morfologia das partículas foi analisada por microscopia eletrônica de varredura (MEV) em um microscópio eletrônico de varredura da PHILIPS modelo ESEM da marca Philips. As análises de microscopia eletrônica de transmissão (MET) foram realisadas em um microscópio eletrônico de transmissão da Philips-200 kV com acessório de análise química por energia dispersiva (EDS). Para a determinação do teor de carbono e molibdênio do carbeto preparado, foram utilizadas a técnica de análise elementar e espectrometria de emissão atômica (ICP-AES), respectivamente. A análise elementar foi realizada no CHN 2400 da Perkin-Elmer e a espectrometria de emissão atômica com plasma induzido foi realizada no equipamento Spectro Ciros CCD.

\section{RESULTADOS E DISCUSSÕES}

A Figura 2 apresenta os resultados do refinamento de estrutura nos padrões de difração segundo método de Rietveld (1967). As curvas em preto são os difratogramas experimentais e as curvas representadas em vermelho são os resultados obtidos a partir do programa de refinamento.

Os resultados obtidos no refinamento podem ser visualizados nas Tabelas 1 e 2 . A Tabela 1 apresenta informações sobre a composição das fases presentes em cada temperatura mostrando que a reação de decomposição-redução-carbonetação ocorre de acordo com a Equação 1:

$$
\mathrm{MoO}_{3} \rightarrow \mathrm{Mo}_{4} \mathrm{O}_{11(\mathrm{Pn} 2 \mathrm{la})} \rightarrow \mathrm{MoO}_{2} \rightarrow \mathrm{Mo} \rightarrow \mathrm{Mo}_{4} \mathrm{O}_{11(\mathrm{Pnma})} \rightarrow \mathrm{Mo}_{2} \mathrm{C}
$$

Já a Tabela 2 apresenta o tamanho de cristalito de cada fase. A $660^{\circ} \mathrm{C}$ por 3 horas de reação é obtido o $\mathrm{Mo}_{2} \mathrm{C}$ puro e à medida que se aumenta a temperatura da reação, o tamanho dos cristalitos também aumenta, de acordo com a Tabela 2. Quanto maior a temperatura da redução carbonetação maior o tamanho dos cristalitos da fase $\mathrm{Mo}_{2} \mathrm{C}$, que para a melhor condição de síntese ficou em torno de $24 \mathrm{~nm}$. $\mathrm{O} \mathrm{Mo} 2 \mathrm{C}$ apresentou rede ortorrômbica e seus parâmetros foram: $\mathrm{a}=4.7377 \AA, \mathrm{b}=6.0125 \AA$, $\mathrm{c}=5.2189 \AA$ e $\beta=148.705^{\circ}$. 

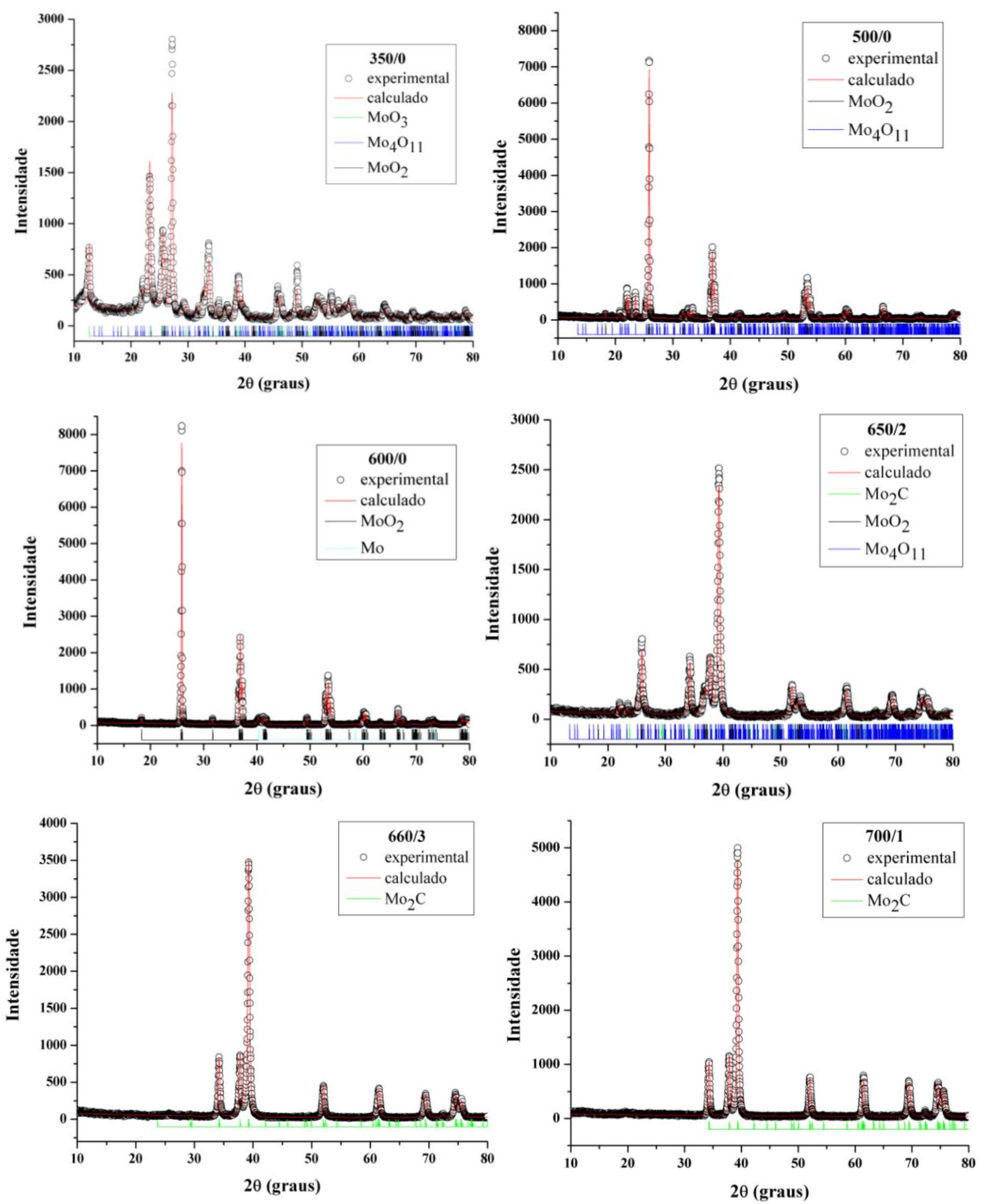

Figura 2 - Refinamento de estrutura aplicado nos padrões de difração para o estudo da decomposição-redução-carbonetação do precursor. 
Tabela 1 - Composição das fases presente obtido pelo refinamento de estrutura

\begin{tabular}{ccccccc}
\hline \multirow{2}{*}{$\begin{array}{c}\text { Temp./tempo } \\
\text { de isoterma } \\
{ }^{\circ} \mathrm{C} / \mathrm{min}\end{array}$} & \begin{tabular}{c}
$\mathrm{MoO}_{3}$ \\
\cline { 2 - 7 }
\end{tabular} & $\begin{array}{c}\mathrm{Mo}_{4} \mathrm{O}_{11} \\
(\mathrm{Pbnm})\end{array}$ & $\begin{array}{c}\mathrm{MoO}_{2} \\
(\mathrm{Pn} 21 \mathrm{a})\end{array}$ & $\begin{array}{c}\mathrm{Mo} 21 / \mathrm{c}) \\
(\mathrm{Im} 3 \mathrm{~m})\end{array}$ & $\begin{array}{c}\mathrm{Mo}_{4} \mathrm{O}_{11} \\
(\mathrm{Pnma})\end{array}$ & $\begin{array}{c}\mathrm{Mo}_{2} \mathrm{C} \\
(\mathrm{Pbcn})\end{array}$ \\
\hline $350 / 0$ & 80,6 & 17,6 & 1,8 & - & - & - \\
$500 / 0$ & - & 25,7 & 74,3 & - & - & - \\
$600 / 0$ & - & - & 94,5 & 5,5 & - & - \\
$650 / 0$ & - & - & 74,9 & 19,7 & - & 5,4 \\
$650 / 60$ & - & - & 25,4 & 24,9 & 4,2 & 45,6 \\
$650 / 120$ & - & - & 27,5 & - & 2,4 & 70,1 \\
$660 / 180$ & - & - & - & - & - & 100,0 \\
$675 / 120$ & - & - & - & - & - & 100,0 \\
$700 / 0$ & - & - & 37,9 & 50,4 & - & 11,6 \\
$700 / 60$ & - & - & - & - & - & 100,0 \\
\hline
\end{tabular}

Tabela 2 - Tamanho dos cristalitos das fases obtidas em cada tempo e temperatura

\begin{tabular}{ccccccc}
\hline \multirow{2}{*}{$\begin{array}{c}\text { Temp./tempo } \\
\text { de isoterma } \\
{ }^{\circ} \mathrm{C} / \mathrm{min}\end{array}$} & $\begin{array}{c}\mathrm{MoO}_{3} \\
(\mathrm{Pbnm})\end{array}$ & $\begin{array}{c}\mathrm{Mo}_{4} \mathrm{O}_{11} \\
(\mathrm{Pn} 21 \mathrm{a})\end{array}$ & $\begin{array}{c}\mathrm{MoO}_{2} \\
(\mathrm{P} 21 / \mathrm{c})\end{array}$ & $\begin{array}{c}\mathrm{Mo} \\
(\mathrm{Im} 3 \mathrm{~m})\end{array}$ & $\begin{array}{c}\mathrm{Mo}_{4} \mathrm{O}_{11} \\
(\mathrm{Pnma})\end{array}$ & $\begin{array}{c}\mathrm{Mo}_{2} \mathrm{C} \\
(\mathrm{Pbcn})\end{array}$ \\
\hline $350 / 0$ & 32,0 & - & 60,0 & - & 18,1 & - \\
$500 / 0$ & - & - & 80,0 & - & 73,0 & - \\
$600 / 0$ & - & - & 83,0 & 10,8 & - & - \\
$650 / 0$ & - & - & 88,0 & 23,7 & - & 18,4 \\
$650 / 60$ & - & 57,0 & 21,1 & 21,2 & - & 19,5 \\
$650 / 120$ & - & 34,0 & 28,1 & - & - & 19,9 \\
$660 / 180$ & - & - & - & - & - & 24,0 \\
$675 / 120$ & - & - & - & - & - & 27,0 \\
$700 / 0$ & - & - & 90,0 & 32,0 & - & 36,0 \\
$700 / 60$ & - & - & - & - & - & 28,5 \\
\hline
\end{tabular}

As Figuras 3 a 5 mostram as micrografias obtidas por MEV dos resíduos obtidos após a decomposição-redução-carbonetação. A morfologia das partículas na sua maioria apresentaram-se uniformes e bastante aglomeradas, com aspecto morfológico de pequenas placas, algumas interligadas e outras não. À medida que aumenta a temperatura de obtenção do carbeto de molibdênio, o tamanho de partículas também aumenta como comprovado pelo refinamento (Tabela 2) e evidenciado nas 


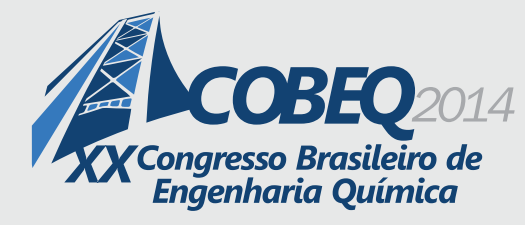

19 a 22 de outubro de 2014

Florianópolis/SC

Figuras 4 e 5. De acordo com a Figura 4 b) observa-se que o carbeto de molibdênio obtido na temperatura de $660^{\circ} \mathrm{C}$ por 180 minutos apresentou partículas em nível de microestruturas formadas a partir de agregados de pequenas partículas.
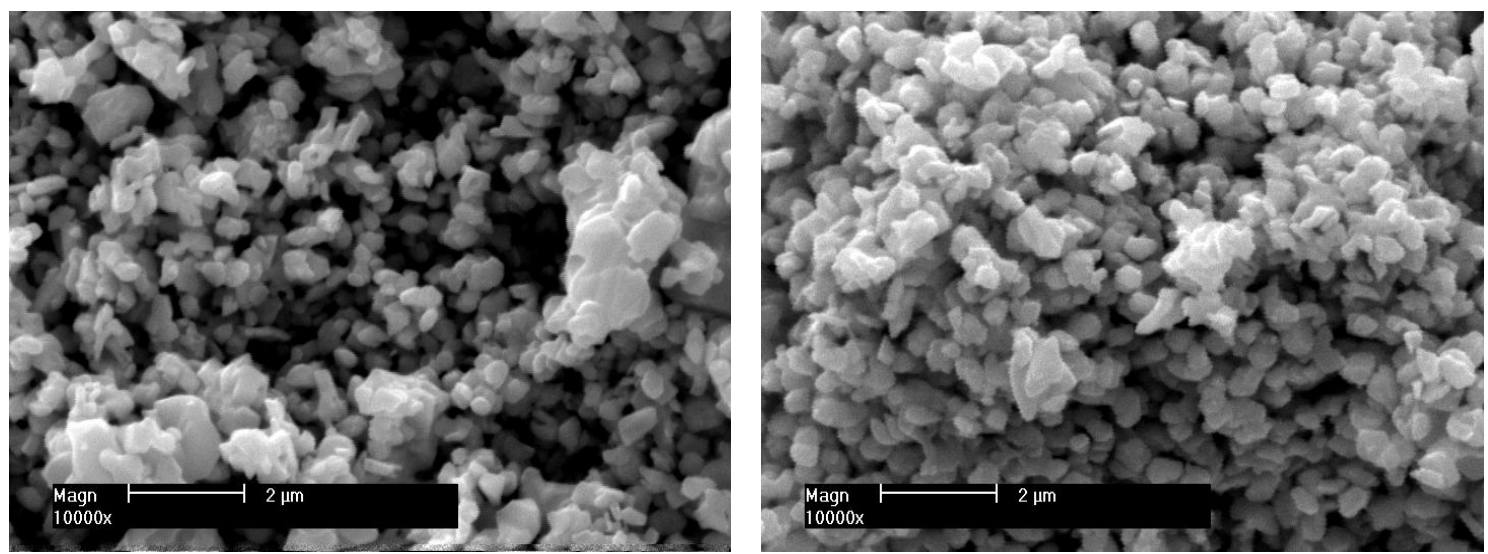

Figura 3 - MEV do precursor tratado: a) $500^{\circ} \mathrm{C} / 0$; b) $600^{\circ} \mathrm{C} / 0$. Aumento de $1000 \mathrm{x}$.
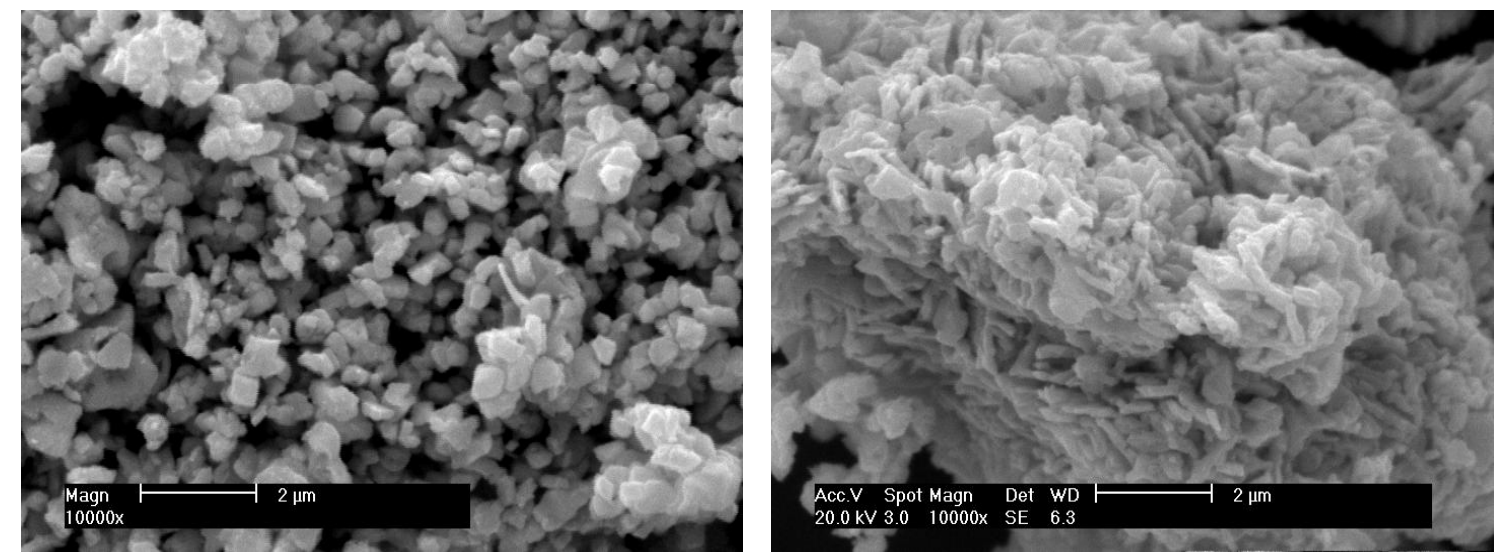

Figura 4 - MEV do precursor tratado: a) $650^{\circ} \mathrm{C} / 1$; b) $660^{\circ} \mathrm{C} / 3$. Aumento de $1000 \mathrm{x}$.
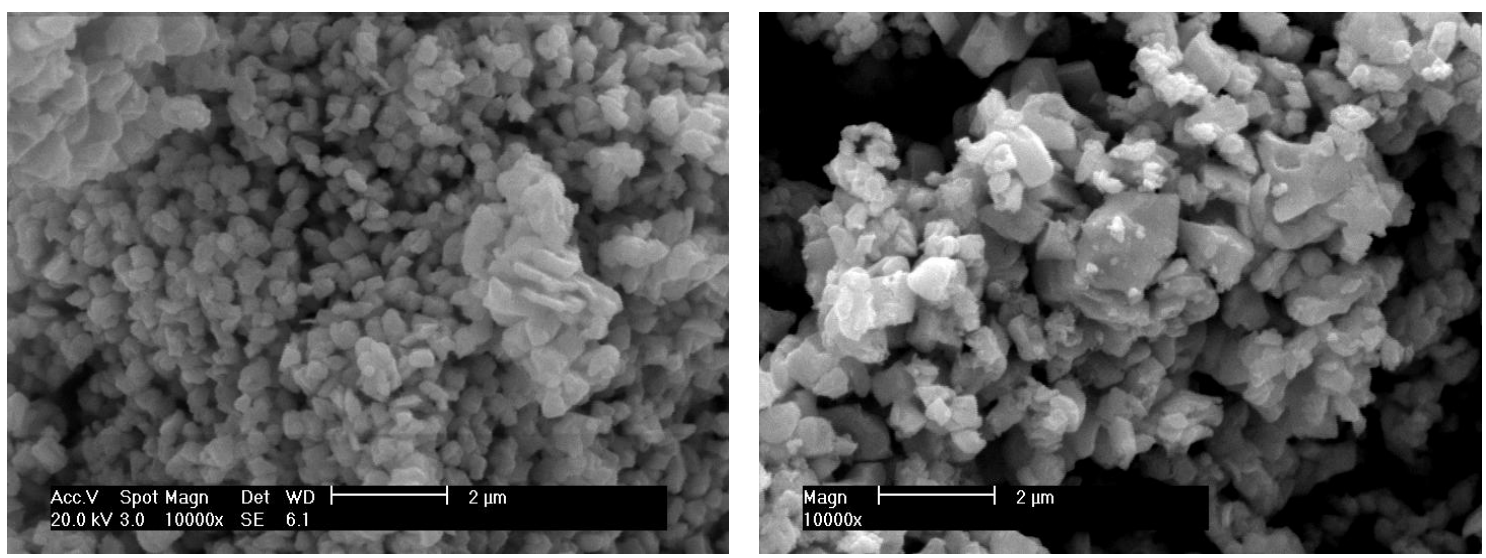

Figura 5 - MEV do precursor tratado: a) $675^{\circ} \mathrm{C} / 2$; b) $700^{\circ} \mathrm{C} / 1$. Aumento de $1000 \mathrm{x}$. 
A Figura 6 mostra a análise de microscopia eletrônica de transmissão do carbeto de molibdênio obtido a $660^{\circ} \mathrm{C}$ por 3 horas de isoterma. Observa-se que o carbeto obtido nesta temperatura apresentase bastante cristalino, onde é possível identificar os planos cristalinos do material. Além disso é possível identificar uma região amorfa que circula toda a partícula, que pode ser atribuído ao carbono livre ou ao oxido de molibdênio $\left(\mathrm{MoO}_{3}\right)$ proveniente do processo de passivacão.

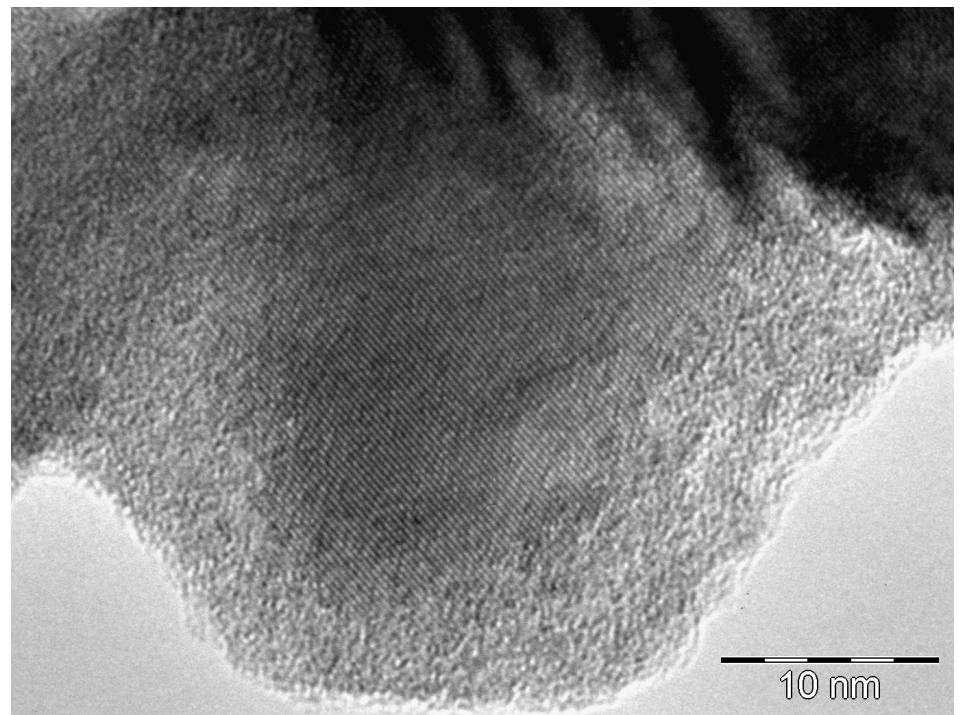

Figura 6 - MET do carbeto de molibdênio obtido a $660^{\circ} \mathrm{C} / 3$ hora.

A quantidade relativa de C/Mo depois da decomposição-redução-carbonetação a $660^{\circ} \mathrm{C}$ por 3 horas seguida da passivacão, está representada na Tabela 3. Verifica-se que a razão molar está muito próximo do valor teórico $(0,5)$ indicando que a quantidade de carbono livre é pequena. Resultados obtidos por Christofoletti et al. (2005) para as temperaturas de $700^{\circ} \mathrm{C}, 800^{\circ} \mathrm{C}$ e $900^{\circ} \mathrm{C}$ mostram uma relação C/Mo após a decomposição-redução-carbonetação de 1,34, 1,75 e 2,52 respectivamente. Este excesso de carbono poderia ser atribuído à presença de fases como $\mathrm{MoC}$ e $\mathrm{MoO}_{2}$ não convertida durante a decomposição-redução-carbonetação (Oshikawa et al., 2001), mas de acordo com o refinamento, o carbeto foi obtido puro a $660^{\circ} \mathrm{C}$ e estas fases não foram identificadas. A baixa temperatura de obtenção do carbeto de molibdênio através do método em estudo favorece a pequena deposição de carbono livre, assim como, o tamanho de cristalito.

Tabela 3 - Análise quantitativa de Mo e C no carbeto de molibdênio obtido a $660^{\circ} \mathrm{C}$ por 3 horas

\begin{tabular}{cccc}
\hline Resultado & $\% \mathrm{Mo}$ & $\% \mathrm{C}$ & $(\mathrm{C} / \mathrm{Mo})^{\mathrm{a}}$ \\
\hline teórico & 94,11 & 5,89 & 0,50 \\
experimental & 93,67 & 6,33 & 0,54 \\
\hline${ }^{\mathrm{a}}$ Razão molar & &
\end{tabular}




\section{CONCLUSÃO}

Através da reação de decomposição-redução-carbonetação do precursor heptamolibdato de amônio o carbeto de molibdênio foi obtido com $24 \mathrm{~nm}$ e uma pequena quantidade de carbono livre e com o refinamento de estrutura pelo método de Rietveld foi possível propor as etapas da reação que representa o processo reacional da síntese do carbeto de molibdênio nanoestruturado.

\section{REFERÊNCIAS}

BEJ, S. K.; BENNETT, C.A.; THOMPSON, L.T. Acid and base characteristics of molybdenum carbide catalysts. Appl. Catal., v. 250, p. 197-208, 2003

CHRISTOFOLETTI, T.; ASSAF, J. M.; ASSAF, E. M. Methane steam reforming on supported and non-supported molybdenum carbides. J. Chem. Eng., v. 106, p. 97-103, 2005.

GU, Y.; LI, Z.; CHEN, L. Synthesis of nanocrystalline $\mathrm{Mo}_{2} \mathrm{C}$ via sodium co-reduction of $\mathrm{MoCl}_{5}$ in benzene. Mater. Res. Bull., v. 38, p. 1119-1122, 2003.

KHABBAZ, S.; HONARBAKHSH-RAOUF, A.; ATAIE, A.; SAGHAFI, M. Effect of processing parameters on the mechanochemical synthesis of nanocrystalline molybdenum carbide. Int. J. Refract. Met. Hard. Mater., v. 41, p.402-407, 2013.

LEVY, R.B. Advanced materials in catalysis, New York: Academic press, 1977.

MEDEIROS, F. F. P.; OLIVEIRA, S. A.; SOUZA, C. P.; SILVA, A. G. P.; GOMES, U. U.; SOUZA, J. F. Synthesis of tungsten carbide through gás-solid reaction at low temperatures. Mat. Sci. Eng. AStruct., v. 315, p. 58-62, 2001.

OSHIKAWA, K.; NAGAI, M.; OMI, S. Characterization of molybdenum carbides for methane reforming by TPR, XRD and XPS. J. Phys.Chem., v. 105, p. 9124-9131, 2001.

PATEL, M.; SUBRAHMANYAM, J. Synthesis of nanocrystalline molybdenum carbide $\left(\mathrm{Mo}_{2} \mathrm{C}\right)$. Mater. Res. Bull., v. 43, p. 2036-2041, 2008.

RIETVELD, H.M. Line profiles of neutron powder-diffraction peaks for structure refinement, Acta Crystallogr., v. 22, p. 151-152, 1967.

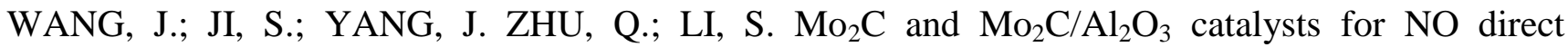
decomposition. Catal. Commun., v. 6, p. 389-393, 2005. 